\title{
The Formation of Abnormal Associations in Schizophrenia: Neural and Behavioral Evidence
}

\author{
Jimmy Jensen ${ }^{1,2}$, Matthäus Willeit', Robert B Zipursky ${ }^{3}$, loulia Savina', Andrew J Smith ${ }^{4}$, Mahesh Menon', \\ Adrian P Crawley ${ }^{5}$ and Shitij Kapur*,1,6 \\ 'Schizophrenia Program and the PET Centre, Centre for Addiction and Mental Health, Toronto, ON, Canada; ${ }^{2}$ Department of Psychiatry, Ullevål \\ University Hospital and Oslo University, Oslo, Norway; ${ }^{3}$ Department of Psychiatry and Behavioural Neurosciences, McMaster University, \\ Hamilton, ON, Canada; ${ }^{4}$ Department of Psychology, McMaster University, Hamilton, ON, Canada; ${ }^{5}$ Department of Medical Imaging, University \\ of Toronto, Toronto, ON, Canada; ${ }^{6}$ Department of Psychiatry, University of Toronto, Toronto, ON, Canada
}

\begin{abstract}
It is hypothesized that due to an abnormal functioning of the reward system patients with schizophrenia form context-inappropriate associations. It has been shown that the dopamine target regions, especially the ventral striatum, are critical in the formation of reward associations. We wanted to examine how the ventral striatum responds as patients learn reward-related associations and how this neural response is linked to objective and subjective behavioral measures. Functional magnetic resonance imaging (fMRI) Blood oxygen level dependent (BOLD) responses were examined using aversive Pavlovian learning in 13 medicated patients with schizophrenia and 13 matched healthy controls. Colored circles served as conditioned stimulus (CS + ) while a loud, individually adjusted, noise served as the unconditioned stimulus. Circles of another color served as neutral comparators (CS-). Subjective indices were assessed by a post-scan self-report, and galvanic skin responses (GSR) were used as objective measures of associative learning. fMRI data were analyzed using a random effects model in SPM2. Patients showed inappropriately strong activations in the ventral striatum in response to the neutral stimulus (CS-) as compared to the healthy controls. Consistent with this neural evidence of aberrant learning, patients also showed evidence of abnormal learning by self-report and as indexed by GSR. The main finding here is that patients with schizophrenia, when exposed to neutral stimuli in a threatening situation, show an abnormal pattern of learning. The aberrant activations and response are consistent with the idea that patients aberrantly assign motivational salience to neutral stimuli, and this process may be one of the aberrations that predisposes them to psychosis.
\end{abstract}

Neuropsychopharmacology (2008) 33, 473-479; doi: I 0. I038/sj.npp. I 301437; published online 2 May 2007

Keywords: schizophrenia; $\mathrm{MRR}$; striatum; learning; reward; salience

\section{INTRODUCTION}

It has been proposed that delusions, which are a hallmark of psychosis in schizophrenia, are the product of abnormally reinforced/rewarded thoughts and associations. Under normal circumstances the dopamine neurons fire in a context-appropriate, event-linked fashion and this facilitates the formation of new associations, and strengthens already learnt associations (King et al, 1984). It is hypothesized that in schizophrenia the dopamine system is dysregulated leading to a 'chaotic' firing of dopamine neurons and heightened levels of dopamine release (King et al, 1984; Shaner, 1999). As a result, event-independent

* Correspondence: Dr S Kapur, Schizophrenia Program and the PET Centre, Centre for Addiction and Mental Health, 33 Russell St, Toronto, ON, Canada, Tel: 416979 6890,

E-mail: shitij_kapur@camh.net

Received 27 November 2006; revised 26 March 2007; accepted 28

March 2007 and context-inappropriate associations are reinforced leading to the formation of fixed and false associations (delusions) (Kapur, 2003; King et al, 1984; Miller, 1984; Shaner, 1999).

The formation and expression of these associations involves the 'brain reward system', with a central role for the ventral striatum (nucleus accumbens in animals) - a region that receives information-carrying inputs from the prefrontal cortex and amygdala and dopaminergic inputs from the midbrain. Several recent studies in normal volunteers have shown a significant role of the ventral striatum in the learning of associations - both appetitive as well as aversive (eg Jensen et al, 2007; McClure et al, 2003; O'Doherty et al, 2003, 2004). These studies show that as associations are learnt, structures that are initially activated by unconditioned stimuli, come to respond to neutral stimuli as a function of their repeated pairings. It has been suggested that this process may be abnormal in schizophrenia (Kapur, 2003; King et al, 1984; Shaner, 1999), thus leading to the learning and reinforcement of inappro- 
priate associations. Pharmacological manipulation of the dopamine system affects the Blood oxygen level dependent (BOLD) activity that occurs during learning in instrumental reward learning (Pessiglione et al, 2006) and in aversive conditioning (Menon et al, in press). Menon et al used event related functional magnetic resonance imaging (fMRI) and an aversive conditioning paradigm and showed that amphetamine increases a learning related signal in striatal regions to cues signaling aversive events, while haloperidol eliminates this signal thus suggesting that dopamine modulates the BOLD responses to aversive stimuli in an aversive conditioning paradigm. It is interesting in that regard that the medical treatment of psychosis involves antipsychotics, drugs which have been shown to block the dopamine system within the ventral striatum (and nucleus accumbens in animals) leading to profound molecular and physiological changes in the brain reward circuits (Arnt and Skarsfeldt, 1998; Wadenberg et al, 2001).

Despite this emerging body of evidence linking the ventral striatum to the formation of rewarding associations, only a few reports have considered this implication for schizophrenia. A recent study (Juckel et al, 2006b) reported that unmedicated patients with schizophrenia showed reduced ventral striatal activation during presentation of reward-predicting cues. Another study focusing on the effect generated by the rewarding experience rather than reward anticipation, showed that patients rated the hedonic value of the pleasant odors lower than controls and also failed to activate limbic/paralimbic structures invoked by normal controls (Crespo-Facorro et al, 2001). Studies using positive and negative pictures as rewarding stimuli also showed that patients failed to recruit parts of a rewardrelated circuit (ventral striatum, amygdala and the orbitofrontal cortex), which were recruited in controls (Paradiso et al, 2003; Taylor et al, 2002, 2005).

While these studies do make a case for some kind of a reward abnormality in schizophrenia (eg Juckel et al, 2006b), they did not obtain any online objective measures of reward-learning, which could link the behavior and brain dysfunction to the disease. Therefore, in this study, we wanted to investigate whether patient's with schizophrenia showed aberrant learning when exposed to motivationally salient (aversive in this case) stimuli. We wanted to relate the neural BOLD findings to objective measures of learning galvanic skin responses (GSR) and subjective reports. Further, to avoid possible performance differences between patients and controls we used a simple Pavlovian conditioning paradigm. We hypothesized that patients would form abnormal associations, either displaying a decreased activation to the CS + or an increased activation to the CSresulting in an abnormal distinction between CS + and CS-, and that these neural abnormalities will be associated with GSR and subjective deficits.

\section{METHODS}

\section{Subjects}

Eighteen patients with schizophrenia and 18 matched healthy controls gave written informed consent according to the guidelines of the Human Subjects Review Committee of the University of Toronto and completed the study. In an initial separate session before the scanning all subjects underwent interviews concerning their physical and psychiatric health history.

Inclusion criteria for patients were: (i) age 18-65; (ii) have a DSM-IV diagnosis of schizophrenia or schizoaffective disorder in the absence of a current major depressive or manic episode; (iii) accepting of care on a voluntary basis and capable of consenting to participation in the research study; (iv) able to undergo fMRI with a duration of approximately $50 \mathrm{~min}$. Exclusion criteria for patients were: (i) serious, unstable medical illness or any concomitant major medical or neurological illness; (ii) acute suicidal and/or homicidal ideation; (iii) DSM-IV substance dependence (except caffeine and nicotine) within 3 months before entering the study; (iv) current major depressive episode or a manic episode; (v) metal implants or cardiac pacemaker; and (vi) used any illegal psychoactive drugs in the last two weeks or any painkillers or alcohol in the last $48 \mathrm{~h}$. The criteria for control subjects were similar except that they never had suffered from any psychiatric illness. The patients and healthy subjects were matched on age, gender, education, and general cognitive estimates (Table 1).

Table I Group Description (Mean \pm SD)

\begin{tabular}{|c|c|c|}
\hline & $\begin{array}{c}\text { Patients } \\
N=13\end{array}$ & $\begin{array}{c}\text { Controls } \\
N=13\end{array}$ \\
\hline Age (years) ${ }^{a}$ & $37.6 \pm 8.5$ & $36.5 \pm 11.8$ \\
\hline Gender (no. of females) ${ }^{a}$ & 3 & 4 \\
\hline Education (years) ${ }^{\mathrm{a}}$ & $15.5 \pm 1.7$ & $16.8 \pm 2.6$ \\
\hline WAIS information ${ }^{\mathrm{a}}$ & $21.2 \pm 2.9$ & $22.0 \pm 3.3$ \\
\hline Duration of illness (years) & $13.0 \pm 8.9$ & \\
\hline Clinical global impression & $3.6 \pm 1.3$ & \\
\hline PANSS positive & $\mid 4.1 \pm 7.1$ & \\
\hline PANSS negative & $15.8 \pm 6.4$ & \\
\hline PANSS general & $31.3 \pm 10.5$ & \\
\hline PANSS total & $61.4 \pm 20.6$ & \\
\hline \multicolumn{3}{|l|}{ Medication } \\
\hline No. I & Olanzapine ( 7.5 mg) & \\
\hline No. 2 & Olanzapine (I 5.0 mg) & \\
\hline No. 4 & Olanzapine ( 10 mg) & \\
\hline No. 6 & Risperidone ( 1.5 mg) & \\
\hline No. 8 & $\begin{array}{c}\text { Olanzapine (22.5 mg) } \\
\text { Haloperidol ( } 2 \mathrm{mg})\end{array}$ & \\
\hline No. 9 & Olanzapine (25 mg) & \\
\hline No. 10 & Risperidone (6.5 mg) & \\
\hline No. 11 & Quetiapine (200 mg) & \\
\hline No. 13 & Olanzapine ( 10 mg) & \\
\hline No. 14 & Olanzapine (20 mg) & \\
\hline No. 16 & Chlorpromazine (100 mg) & \\
\hline No. 17 & Olanzapine (10 mg) & \\
\hline No. 18 & Clozapine (100 mg) & \\
\hline
\end{tabular}

Five of the patients were also prescribed a benzodiazepine.

a No significant differences between patients and healthy controls. 


\section{Experimental Protocol}

fMRI BOLD responses were examined in a classical passive Pavlovian learning paradigm involving aversive events randomly mixed with neutral events. The aversive events consisted of an $800 \mathrm{~ms}$ long loud fire-truck horn burst as unconditioned stimuli (US). The intensity of the US was individually titrated to where it was reported to be 'unpleasant but tolerable'. Colored circles, which preceded the US by $5 \mathrm{~s}$, served as conditioned stimuli $(\mathrm{CS}+)$ and predicted the US according to a $50 \%$ partial reinforcement schedule. Circles of another color that served as neutral comparators (CS-) were followed by a visual star in $50 \%$ of the events. Between trials a fixation cross was displayed for $9 \mathrm{~s}$. Forty CS + and $40 \mathrm{CS}$ - trials were presented during the $18 \mathrm{~min}$ of the experimental session.

\section{Apparatus}

The subjects used an adjustable mirror located above their eyes to view the projected CS images on a screen placed at the foot of the scanner bed. Sound stimuli were delivered by the Commander XG FMRI Audio system (Resonance Technology Inc., Northridge, CA). The E-prime software (Psychology Software Tools, Inc., Pittsburg, PA) controlled the stimulus presentations. GSR were continuously monitored by PowerLab 2/20 (AD Instruments; Castle Hill, Australia) via long well-isolated rf-filtered cables through a wave guide. MRI compatible $\mathrm{Ag} / \mathrm{AgCl}$ electrodes attached to the terminal phalanx on the left index and middle finger respectively were used.

\section{Image Acquisition}

MRI scans were acquired by a GE Signa $1.5 \mathrm{~T}$ scanner (General Electric, Waukesha, WI) equipped with a standard head coil. In a single session, 509 volumes (28 contiguous axial 4.4-mm-thick slices) covering the whole brain were acquired using a $\mathrm{T} 2 *$-sensitive spiral in-out sequence $\left(\mathrm{TR}=2240 \mathrm{~ms} ; \mathrm{TE}=40 \mathrm{~ms}\right.$; flip angle $85^{\circ}$; matrix $64 \times 64$; FOV $200 \times 200 \mathrm{~mm}$ ). This sequence was chosen since it has reduced susceptibility dropout in sensitive areas (Glover and Thomason, 2004) including the ventral striatum. The first three volumes were discarded to allow for $\mathrm{T}_{1}$ equilibrium effects, and the data from the remaining 506 volumes were used in the analysis. For localization purposes, IR-Prepped 3D FSPGR T1-weighted anatomical images (120 contiguous axial 1.1-mm-thick slices) were acquired $\left(\mathrm{TR}=12 \mathrm{~ms} ; \mathrm{TE}=5.4 \mathrm{~ms}\right.$; flip angle $20^{\circ}$; matrix $256 \times 256$; FOV $200 \times 200 \mathrm{~mm}$ ).

\section{Data Quality}

The images were visually inspected for signal dropout due to magnetic susceptibility in the region of ventral striatum. The visual inspection revealed no signal dropout in this region. fMRI data from five patients and five controls could not be used: six scans were lost due to unsuccessful reconstruction or wrongly prescribed scanning parameters; one subject's structural scan indicated brain abnormalities and; three subjects moved more than the allowed $3 \mathrm{~mm}$ during the scan. Of the remaining 13 patients and 13 healthy controls, GSR data could not be obtained for three of the patients and two of the controls due to technical problems.

\section{SPM2 analysis (http://www.fil.ion.ucl.ac.uk/spm)}

All volumes were realigned to the first volume (Friston et al, 1995) and the anatomical image was coregistered to the mean functional image to ensure that they were aligned. The images were spatially normalized (Friston et al, 1995) to a standard EPI template (Evans et al, 1993), resampled at $3 \times 3 \times 3 \mathrm{~mm}$ and smoothed using a $10 \mathrm{~mm}$ full-width halfmaximum (FWHM) isotropic kernel. Data were high-pass filtered using a cutoff value of $128 \mathrm{~s}$. The data were analyzed by modeling four event types as stick functions convolved with a synthetic HRF. The four events consisted of the CS onsets, modelling the CS + followed by the US and the CS + not followed by the US separately as well as modelling CSfollowed by a visual star and the CS - not followed by anything separately. The two regressors consisting of CS followed by US and a visual star were not used in any contrasts. For every subject three contrasts were specified: $\mathrm{CS}+, \mathrm{CS}-$ and $\mathrm{CS}+v s \mathrm{CS}-$. The individual contrast images were moved up to second-level random effects models. The between group random effects models investigated the effects of $\mathrm{CS}+, \mathrm{CS}-$ and $\mathrm{CS}+v s \mathrm{CS}-$ respectively. For the healthy controls, we also made a within-group analysis to establish that the contrast [CS $+v s$ CS-] yielded activations in the ventral striatum similar to previous studies (Jensen et al, 2003). The data were thresholded at FDR $<0.05$ except for the CS $+v s$ CS comparisons where small volume correction for regions of interest (ventral striatum) were used based on coordinates from a previous study (Jensen et al, 2003). We used spheres with a radius of $9 \mathrm{~mm}$ centered on MNI coordinates $-8,10$, -2 for the left ventral striatum and $12,6,-4$ for the right ventral striatum.

\section{GSR Recording and Analysis}

The GSR was sampled at $10 \mathrm{~Hz}$. To correct for possible MRI induced artifacts the GSR signal was digitally low-pass filtered using a cutoff value of $1 \mathrm{~Hz}$. To determine the GSR, the peak amplitude value within the $10 \mathrm{~s}$ following the cue onset was taken and subtracted by the value at the CS onset. The frequency of values higher than $0.05 \mu \mathrm{S}$ (Boucsein, 1992) was calculated for each of the four trial types modeled as above. For comparisons, Student's $t$-tests for related samples and repeated measures analysis of variance were performed.

\section{Self Reports}

Using a post-scan questionnaire, the subjects were asked to rate their degree of uneasiness when the CSs were shown. This was done on scales with four anchors (relaxed to extremely uneasy). In comparisons we used rank-order Wilcoxon test.

\section{Correlations}

Pearson or Spearman correlations of BOLD ( $\beta$ values in the ventral striatum) and symptoms (PANSS positive and 
PANSS negative), and medications were performed depending on distribution of data. For the medication correlation we used the dose from the eight subjects that were given Olanzapine instead of trying to convert all drugs to a standard drug.

\section{Data Handling}

For BOLD FMRI, and GSR data only the non-reinforced trials for both CS + and CS- were used in contrasts.

\section{RESULTS}

Data from 13 medicated stable patients with schizophrenia and 13 matched healthy controls were analyzed. Group descriptions are displayed in Table 1.

Patients showed evidence of abnormal learning by selfreport (Figure 1). The patients could not distinguish between CS + vs CS $-[0.50$ vs $0.25 ; Z=1.13, p=\mathrm{NS}]$ while the control subjects reported a clear subjective aversive response viewing the CS + as compared to CS $-[1.15$ vs $0.08 ; Z=2.40, p<0.01$.

GSR, an autonomic measure of the level of arousal, were continuously recorded during the fMRI session and used as objective indices of conditioned responses. While both patients $(p<0.05)$ and healthy controls $(p<0.001)$ displayed more GSR above threshold to the CS + as compared to CS-, a group $\times$ condition interaction $[\mathrm{F}(1,19)=10.92 ; p<0.01]$ was obtained suggesting that patients showed less GSR above threshold to CS + but more GSR to CS - stimuli compared to controls (Figure 1). This is in keeping with the self-reported findings.

In the PMRI BOLD data we wanted to examine differences in responses to the CS + and CS - between the patients and the controls. No differences between the groups in responses to the CS + were obtained in any region of the brain after correcting for multiple comparisons. For the CS-, an activation in the ventral striatum (coordinate 6,3 , $0 ; Z=4.14$; Figure 2) was obtained for the patients with schizophrenia compared to the controls after correcting for multiple comparisons $\left(p_{\mathrm{FDR}}<0.05\right)$. Clusters surviving corrections are reported in Table 2. There were no activations in the striatum or in any other brain region that were stronger among controls compared to the patients for the CS- condition after correction for multiple comparisons.

A one-sample $t$-test was performed in the healthy controls showing an activation in the ventral striatum for the contrast $[\mathrm{CS}+>\mathrm{CS}-]$ at peak coordinate $6,9,0(Z=2.92$; $p<0.05)$. Two-sample $t$-tests were used to test for differences in brain activations for the CS $+v s$ the CSbetween the patients with schizophrenia and the healthy controls. Searching a small volume centered at previously reported coordinates (Jensen et al, 2003), an activation in the ventral striatum (peak at coordinate $-6,12,3 ; Z=3.08$; $p<0.05)$ for the healthy controls as compared to the patients were obtained (Figure 3). An examination of the $\beta$ values (Figure 3 ) showed that this difference was driven by a much larger response to the CS- among the patients while both patients and controls had similar responses to CS +. To examine the generalizability of the results, we analyzed the data using a small volume search centered on the coordinates reported by Juckel et al (coordinate -15, 11, -6) (Juckel et al, 2006b) which again yielded the same findings, that is significant peak activation at co-ordinate $-6,12,3$.

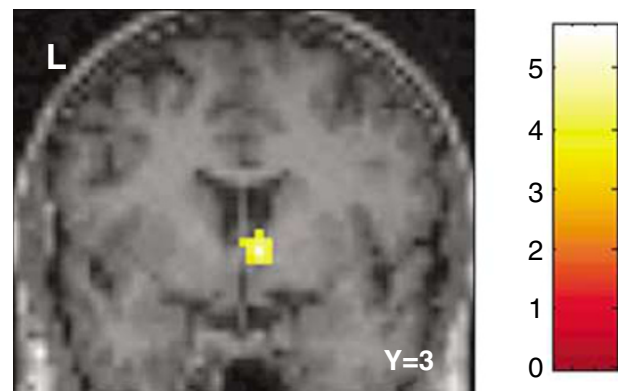

Figure 2 A statistical parametric map showing the significantly larger effect in the ventral striatum in patients with schizophrenia compared to healthy controls for the CS-. The colors refer to $t$-values. $N=13$ in each group.

Table 2 Activated Regions in Response to CS-; Patients > Controls

\begin{tabular}{lccc}
\hline Region & Co-ordinate & (No. voxels) & Z-value \\
\hline Right ventral striatum & $6,3,0$ & 78 & 4.14 \\
Middle cingulate cortex & $-6,-36,39$ & 521 & $4.5 \mid$ \\
Right thalamus & $12,-30,3$ & 458 & 4.42 \\
Right prefrontal cortex & $36,45,-3$ & 67 & 4.71 \\
Right Hippocampus & $15,-21,-15$ & 66 & 3.79 \\
\hline
\end{tabular}

Corrected for multiple comparisons FDR $<0.05$.
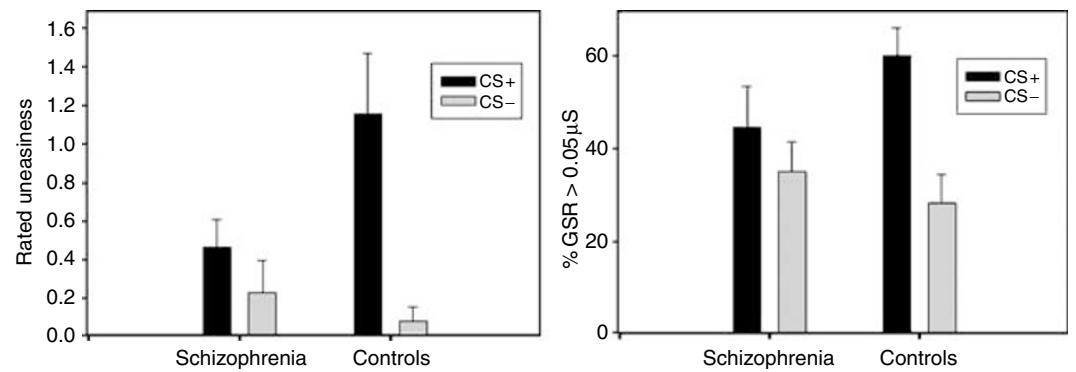

Figure I The left panel shows the subjective ratings of uneasiness for the conditions. The right panel displays the frequency of galvanic skin responses above threshold (ie $>0.05 \mu \mathrm{S}$ ) for the conditions. The error bars refer to SE. 

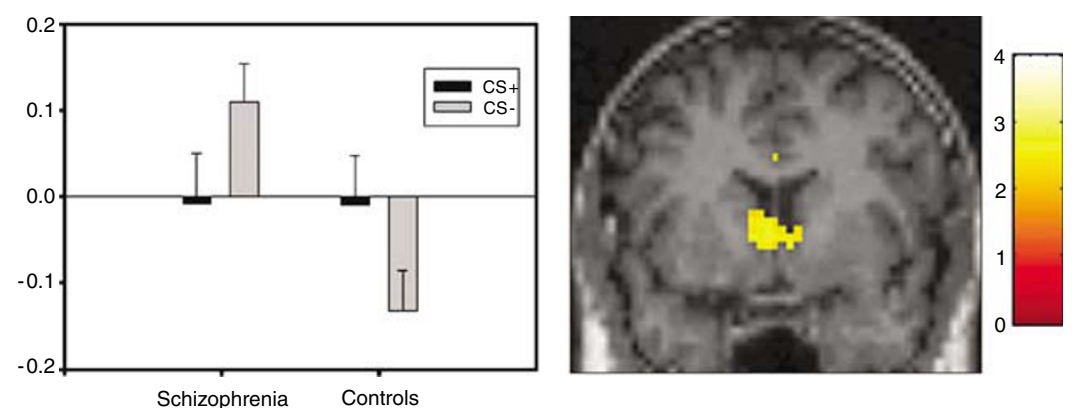

Figure 3 The left panel shows the $\beta$ values for the conditions in the left ventral striatum (at coordinates $(-6,12,3)$ where the error bars refer to SE. The right panel is a statistical parametric map in the ventral striatum $(Y=12)$ for the contrast $(C S+>C S-)$ in healthy controls compared to patients with schizophrenia. The figure suggests that the patients have a response above the mean to the CS- while healthy controls are under the mean. In this voxel the response to CS + is similar in the groups. The colors refer to $t$-values.

No significant correlations between symptoms (PANSS positive, $N=13 ; r=0.18 ; p=\mathrm{NS}$, or PANSS negative, $N=13 ; r=-0.37 ; p=\mathrm{NS})$ or medication dose $(N=8$; $\rho=-0.17 ; p=\mathrm{NS})$ and BOLD responses for CS + were obtained among the patients. Neither were there any associations between symptoms (PANSS positive, $N=13$; $r=0.10 ; p=\mathrm{NS}$, or PANSS negative, $N=13 ; r=-0.13$; $p=\mathrm{NS})$ or medication dose $(N=8 ; \rho=-0.10 ; p=\mathrm{NS})$ and BOLD responses for CS-. Exploratory correlation analyses were done to investigate if there were any associations between self reported uneasiness to CS-, GSR to the CS-, and BOLD responses to the CS- in the ventral striatum among the patients. No significant associations were obtained.

\section{DISCUSSION}

The main finding here is that patients with schizophrenia, when exposed to neutral stimuli in a threatening situation, show an abnormal pattern of learning. Their ability to distinguish (subjectively as measured with self report and at a physiological level as measured with GSR) between neutral stimuli (CS - ) and stimuli truly linked to danger or aversive outcomes $(\mathrm{CS}+)$ is impaired. Further, neutral stimuli come to be associated with significant conditioned brain activations among the patients - an abnormality that was accompanied with abnormal physiological (GSR) and explicit learning (by self-report).

The ventral striatum plays a central role in reward processing and it has been suggested that activations of the ventral striatum normally mediate the incentive or motivational salience of environmental stimuli (Jensen et al, 2003, 2007; Zink et al, 2003, 2004, 2006). The stronger responses to the neutral stimulus among patients may reflect an aberrant attribution of motivational salience to neutral stimuli (Kapur, 2003). As a result, stimuli that are neutral and innocuous among healthy controls may gain a status as motivationally salient among patients with schizophrenia. In line with this finding, a recent study using a word classification paradigm showed that deluded patients with schizophrenia displayed a bias towards classifying words as unpleasant, a misattribution of salience, compared to nondeluded patients and healthy controls (Holt et al, 2006b). It has been proposed that positive symptoms (such as delusions and hallucinations) are the product of abnormally reinforced thoughts and associations, and the current study could serve as a model of how context-inappropriate associations are reinforced (Kapur, 2003; King et al, 1984; Miller, 1984; Shaner, 1999).

However, a number of caveats should be added to such a proposal-First, ours is a cross-sectional study in patients who had pre-existing delusions and thus we are unable to resolve the causal contribution of these aberrant associations. Second, we did not find a reliable association between the psychotic symptoms and aberrant activations, though this may have been limited by the small sample size and the fact that the antipsychotics had muted the psychosis. Also, the presence of the antipsychotic medication introduces a possible confound that the aberrant pattern seen may be a product of the dopamine block introduced by antipsychotics. Recently it was shown that patients treated with atypical antipsychotics activated the ventral striatum whereas patients on typical antipsychotics failed to recruit this region during a monetary incentive task (Juckel et al, 2006a). It should be noted that 11 out of 13 patients in the current study were treated with atypical medication and one patient was prescribed both types suggesting that the formation of abnormal associations might be due to the disease rather than medication. However, to address this question a study using drug-naïve patients must be performed.

The study adds to a growing body of literature relating to reward abnormalities in schizophrenia, although the previous literature does not tell a simple story. Using differing paradigms, some studies find reduced reward learning in conditioning experiments in patients with schizophrenia (Hofer et al, 2001; Kosmidis et al, 1999; Lynn, 1963), others describe enhanced reward learning in schizophrenia (Sears et al, 2000; Spain, 1966), and yet others find no differences between patients and controls (Howe, 1958). Latent inhibition (LI), a model of reward learning that requires subjects to ignore irrelevant stimuli and where learning can be measured objectively using GSR has been used to provide a more objective index of reward learning. Acute unmedicated patients show a disruption of LI (ie evidence for aberrant reward learning) whereas chronic and medicated patients show normal LI (Gray et al, 1997; Vaitl et al, 2002). Most earlier imaging studies on reward in schizophrenia have focused on the affect generated by the rewarding experience rather than reward learning. Patients have been reported to rate the hedonic value of pleasant odors lower 
than controls and fail to activate limbic/paralimbic structures invoked by normal controls (Crespo-Facorro et al, 2001). Studies using positive and negative pictures as rewarding stimuli (Paradiso et al, 2003; Taylor et al, 2002, 2005) also showed that patients failed to recruit parts of reward related circuits. These earlier imaging studies on reward in schizophrenia have focused on the failure to activate structures during reward per se whereas the current study suggest an aberrant activation pattern towards cues predicting neutral stimuli in a reward learning paradigm. In line with the current findings, an elevated activity in hippocampus and amygdala has been shown in schizophrenia during passive viewing of human faces, displaying both neutral and negative emotions (Holt et al, 2006a).

While there are few previous studies that have examined the formation of reward associations using functional neuroimaging, the study that comes closest in its methods and design is by Juckel and collaborators (Juckel et al, 2006b), who found a negative correlation between the severity of negative symptoms and activation of the ventral striatum. In the current study no significant correlations were obtained between activations in the ventral striatum and psychopathological symptoms. A reason for this might be that the patients in the current study were less symptomatic compared to the sample in the study of Juckel et al. A direct comparison with the study by Juckel and collaborators is difficult since they did not report any behavioral data or fMRI $\beta$ values for the different conditions. Also, there are two major differences between the studies: (1) Juckel et al used a monetary paradigm while the current study used primary aversive stimulations and; (2) they used drug-free patients while the patients in the current study all were stable on medications and displayed less psychopathological symptoms. Nonetheless, Juckel et al (2006b) also showed that patients with schizophrenia displayed reduced ventral striatal activation when exposed to reward predicting $v s$ relative neutral cues, a finding that is consistent with our observation that the aberrant distinction between $\mathrm{CS}+$ and $\mathrm{CS}-$ may be driven by abnormal activations in the ventral striatum in response to $\mathrm{CS}$-.

In summary, among patients with schizophrenia neutral stimuli come to be associated with significant conditioned brain activations - an abnormal formation of associations. This neural abnormality was accompanied with changes in physiological measures and subjective reports which support the idea of aberrant conditioning. Future studies should examine whether such abnormalities precede the onset of psychosis and how treatment modifies them.

\section{ACKNOWLEDGEMENTS}

This study was supported by a grant from the Canadian Institutes of Health Research.

\section{DISCLOSURE/CONFLICT OF INTEREST}

The authors declare no personal financial interest that could be perceived as constituting a potential conflict of interest.

\section{REFERENCES}

Arnt J, Skarsfeldt T (1998). Do novel antipsychotics have similar pharmacological characteristics? A review of the evidence. Neuropsychopharmacology 18: 63-101.

Boucsein W (1992). Electrodermal Activity. Planum Press: New York.

Crespo-Facorro B, Paradiso S, Andreasen NC, O'Leary DS, Watkins GL, Ponto LL et al (2001). Neural mechanisms of anhedonia in schizophrenia: a PET study of response to unpleasant and pleasant odors. JAMA 286: 427-435.

Evans AC, Collins DL, Mills SR, Brown ED, Kelly RL, Peters TM (1993). 3D statistical neuroanatomical models from 305 MRI volumes. Proceedings of the Institute of Electrical and Electronics Engineering - Nuclear Science Symposium and Medical Imaging 3: $1813-1817$.

Friston KJ, Holmes AP, Poline JB, Grasby PJ, Williams SC, Frackowiak RS et al (1995). Analysis of fMRI time-series revisited. Neuroimage 2: 45-53.

Glover GH, Thomason ME (2004). Improved combination of spiral-in/out images for BOLD fMRI. Magn Reson Med 51: 863-868.

Gray JA, Moran PM, Grigoryan G, Peters SL, Young AM, Joseph MH (1997). Latent inhibition: the nucleus accumbens connection revisited. Behav Brain Res 88: 27-34.

Hofer E, Doby D, Anderer P, Dantendorfer K (2001). Impaired conditional discrimination learning in schizophrenia. Schizophr Res 51: 127-136.

Holt DJ, Kunkel L, Weiss AP, Goff DC, Wright CI, Shin LM et al (2006a). Increased medial temporal lobe activation during the passive viewing of emotional and neutral facial expressions in schizophrenia. Schizophr Res 82: 153-162.

Holt DJ, Titone D, Long LS, Goff DC, Cather C, Rauch SL et al (2006b). The misattribution of salience in delusional patients with schizophrenia. Schizophr Res 83: 247-256.

Howe ES (1958). GSR conditioning in anxiety states, normals, and chronic functional schizophrenic subjects. J Abnorm Psychol 56: 183-189.

Jensen J, McIntosh AR, Crawley AP, Mikulis DJ, Remington G, Kapur S (2003). Direct activation of the ventral striatum in anticipation of aversive stimuli. Neuron 40: 1251-1257.

Jensen J, Smith AJ, Willeit M, Crawley AP, Mikulis DJ, Vitcu I et al (2007). Separate brain regions code for salience versus valence during reward prediction in humans. Human Brain Mapping 28: 294-302.

Juckel G, Schlagenhauf F, Koslowski M, Filonov D, Wustenberg T, Villringer A et al (2006a). Dysfunction of ventral striatal reward prediction in schizophrenic patients treated with typical, not atypical, neuroleptics. Psychopharmacology (Berlin) 187: 222-228.

Juckel G, Schlagenhauf F, Koslowski M, Wustenberg T, Villringer A, Knutson B et al (2006b). Dysfunction of ventral striatal reward prediction in schizophrenia. Neuroimage 29: 409-416.

Kapur S (2003). Psychosis as a state of aberrant salience: a framework linking biology, phenomenology, and pharmacology in schizophrenia. Am J Psychiatry 160: 13-23.

King R, Barchas JD, Huberman BA (1984). Chaotic behavior in dopamine neurodynamics. Proc Natl Acad Sci USA 81: 1244-1247.

Kosmidis MH, Breier A, Fantie BD (1999). Avoidance learning in schizophrenia: a dissociation between the effects of aversive and non-aversive stimuli. Schizophr Res 38: 51-59.

Lynn R (1963). Russian theory and research on schizophrenia. Psychol Bull 60: 486-498.

McClure SM, Berns GS, Montague PR (2003). Temporal prediction errors in a passive learning task activate human striatum. Neuron 38: 339-346. 
Menon M, Jensen J, Vitcu I, Graff-Guerro A, Crawley AP, Smith MA et al (in press). Temporal difference modeling of the BOLD response during aversive conditioning in humans: effects of dopaminergic modulation. Biological Psychiatry.

Miller R (1984). Major psychosis and dopamine: controversial features and some suggestions. Psychol Med 14: 779-789.

O'Doherty J, Dayan P, Schultz J, Deichmann R, Friston K, Dolan RJ (2004). Dissociable roles of ventral and dorsal striatum in instrumental conditioning. Science 304: 452-454.

O'Doherty JP, Dayan P, Friston K, Critchley H, Dolan RJ (2003). Temporal difference models and reward-related learning in the human brain. Neuron 38: 329-337.

Paradiso S, Andreasen NC, Crespo-Facorro B, O'Leary DS, Watkins GL, Boles Ponto LL et al (2003). Emotions in unmedicated patients with schizophrenia during evaluation with positron emission tomography. Am J Psychiatry 160: 1775-1783.

Pessiglione M, Seymour B, Flandin G, Dolan RJ, Frith CD (2006). Dopamine-dependent prediction errors underpin reward-seeking behaviour in humans. Nature 442: 1042-1045.

Sears LL, Andreasen NC, O'Leary DS (2000). Cerebellar functional abnormalities in schizophrenia are suggested by classical eyeblink conditioning. Biol Psychiatry 48: 204-209.

Shaner A (1999). Delusions, superstitious conditioning and chaotic dopamine neurodynamics. Med Hypotheses 52: 119-123.
Spain B (1966). Eyelid conditioning and arousal in schizophrenic and normal subjects. J Abnorm Psychol 71: 260-266.

Taylor SF, Liberzon I, Decker LR, Koeppe RA (2002). A functional anatomic study of emotion in schizophrenia. Schizophr Res 58: 159-172.

Taylor SF, Phan KL, Britton JC, Liberzon I (2005). Neural response to emotional salience in schizophrenia. Neuropsychopharmaco$\log y$ 30: 984-995.

Vaitl D, Lipp O, Bauer U, Schuler G, Stark R, Zimmermann M et al (2002). Latent inhibition and schizophrenia: pavlovian conditioning of autonomic responses. Schizophr Res 55: 147-158.

Wadenberg ML, Soliman A, VanderSpek SC, Kapur S (2001). Dopamine $\mathrm{D}(2)$ receptor occupancy is a common mechanism underlying animal models of antipsychotics and their clinical effects. Neuropsychopharmacology 25: 633-641.

Zink CF, Pagnoni G, Chappelow J, Martin-Skurski M, Berns GS (2006). Human striatal activation reflects degree of stimulus saliency. Neuroimage 29: 977-983.

Zink CF, Pagnoni G, Martin ME, Dhamala M, Berns GS (2003). Human striatal response to salient nonrewarding stimuli. J Neurosci 23: 8092-8097.

Zink CF, Pagnoni G, Martin-Skurski ME, Chappelow JC, Berns GS (2004). Human striatal responses to monetary reward depend on saliency. Neuron 42: 509-517. 